\title{
Tetragonal states from epitaxial strain on metal films
}

\author{
P. M. Marcus \\ IBM Research Division, Thomas J. Watson Research Center, Yorktown Heights, New York 10598 \\ P. Alippi \\ Fritz-Haber Institut der Max Planck Gesellschaft, Faradayweg 4-6, D-14195 Berlin-Dahlem, Germany
}

(Received 13 March 1997; revised manuscript received 15 May 1997)

\begin{abstract}
The tetragonal states produced by isotropic pseudomorphic epitaxial strain in the (001) plane on a tetragonal phase of a crystal are calculated for $\mathrm{V}, \mathrm{Ti}, \mathrm{Rb}, \mathrm{Li}, \mathrm{K}$, and $\mathrm{Sr}$ from first-principles electronic theory. It is shown that each metal has two tetragonal phases corresponding to minima of the total energy with respect to tetragonal deformations (and hence are equilibrium phases) and that the equilibrium phases are separated by a region of inherent instability. The equilibrium phase for any strained tetragonal state can thus be uniquely identified. Lattice constants and relative energies of the two phases and the saddle point between them are tabulated, as well as the tetragonal elastic constants of each phase. [S0163-1829(97)04944-8]
\end{abstract}

\section{INTRODUCTION}

Metal crystals in the body-centered-tetragonal structure are known to have two total-energy minima as functions of tetragonal lattice constants $a$ and $c .{ }^{1-3}$ The minima are appropriately called equilibrium metallic phases since they persist without applied stress and are stable under small tetragonal deformations. Previous work by the authors ${ }^{4}$ defined and discussed epitaxial Bain paths (EBP's), which are sequences of tetragonal states that include the equilibrium tetragonal phases. These paths give the strained tetragonal states produced by isotropic epitaxial strain, i.e., isotropic two-dimensional biaxial or in-plane strain, imposed on the (001) planes of the equilibrium phases. In the previous work the EBP's for V, Co, and $\mathrm{Cu}$ were found using firstprinciples total-energy calculations and the EBP's were compared with the paths produced by uniaxial stress on the phases, which were called in that work uniaxial Bain paths.

The EBP's were shown to be directly useful in interpreting the bulk structure of epitaxial films determined, for example, by quantitative low-energy electron diffraction (QLEED). A comparison of the measured structure of a film with the states on the EBP's identifies the phase from which the film is produced by the epitaxial strain. Thus a film of Co on $\mathrm{Cu}(001)$ was shown to be strained fcc $\mathrm{Co}$, but a film of $\mathrm{Co}$ on $\mathrm{Fe}(001)$ was shown to be strained body-centeredtetragonal (bct) Co, a metastable phase of Co, whereas bcc Co was shown to be unstable.

In the tetragonal plane, whose coordinates are tetragonal lattice constants, the EBP is a continuous path that passes through the two phase points at the energy minima and through the saddle point of energy between the two minima. It was shown that between the minima a segment of the EBP exists that includes the saddle point, but not the minima, which consists of inherently unstable states. More generally, strained states of each phase, not just those on the EBP, were shown to be separated by a region of inherently unstable tetragonal states, so that an observed strained tetragonal state has a connection to just one equilibrium phase through stable but constrained states.
The present work gives the EBP's for six metals based on the published first-principles calculations for tetragonal structures by Sliwko, Mohn, Schwarz, and Blaha. ${ }^{3}$ The calculation procedures for finding the EBP, the contours of constant energy, and the unstable region are described in Sec. II. The results are described in Sec. III with two tables and four figures. Section IV discusses why the EBP is useful, the significance of the unstable region, and notes defects and generalizations of these calculations.

\section{CALCULATION PROCEDURES}

The calculations of total energy $E$ as a function of the tetragonal lattice parameters $a$, the side of the square cross section, and $c$, the height of the unit cell, used the powerseries expansions given in Ref. 3, whereas the calculations in Ref. 4 used the full-potential augmented plane-wave program WIEN95 directly. The power-series expansions were fitted in Ref. 3 to extensive first-principles calculations in the local-density approximation (LDA) with WIEN95. The expansions give $E$ within specified ranges of $c / a$ and volume per atom $V=c a^{2} / 2$ that include the minima and saddle point and have the form

$$
E=\sum_{i=0}^{n} \sum_{j=0}^{m} A_{i j}(c / a)^{i} V^{j} .
$$

The coefficients $A_{i j}$ are tabulated in Ref. 3 to eight significant figures and are available in electronic form from the authors. In Eq. (1) $n$ is 5 or $6, m$ is 3 , and $E$ is obtained in the specified ranges to an accuracy stated to be better than 0.01 mRy. Some comments on this stated accuracy are made in Sec. IV. A useful feature of formula (1) is that analytical formulas for the first and second derivatives of $E$ may be readily derived.

The EBP for each metal is found by calculating $E$, $(\partial E / \partial c)_{a}$, and $(\partial E / \partial a)_{c}$ as a function of $c$ at constant $a$ and locating the $c$ value for which $E$ has a minimum or, equivalently, locating the zero of $(\partial E / \partial c)_{a}$. The minimum corresponds to the epitaxial film condition of zero normal stress 
on the (001) surface. As $a$ ranges over the structures between the phase points, the EBP is traced by these minima of $E$ at each $a$. Since $E$ and $(\partial E / \partial c)_{a}$ are evaluated easily, a dense grid of $c$ values permits simple interpolation to four significant figures for $c, E$, and $(\partial E / \partial a)_{c}$ at the minimum of $E$ for any $a$.

The two phase points and the saddle point correspond to stationary points for $E$ and hence are located by interpolating on the EBP itself to find points where $(\partial E / \partial a)_{c}$ vanishes along with $(\partial E / \partial c)_{a}$. Two of the three stationary points are always cubic points, since at a cubic point if $(\partial E / \partial c)_{a}=0$ then also $(\partial E / \partial a)_{c}=0$. The contours of constant $E$ are similarly found by interpolating the desired $E$ in a tabulation of $E(c)$ at values of $a$ over a range of $a$ that covers the desired contour.

Tetragonal elastic constants at any $a$ and $c$ may be defined by

$$
\begin{gathered}
\bar{c}_{11} \equiv \frac{a^{2}}{V} \frac{\partial^{2} E}{\partial a^{2}}=2\left(c_{11}+c_{12}\right), \\
\bar{c}_{13} \equiv \frac{a c}{V} \frac{\partial^{2} E}{\partial a \partial c}=2 c_{13}, \\
\bar{c}_{33} \equiv \frac{c^{2}}{V} \frac{\partial^{2} E}{\partial c^{2}}=c_{33} .
\end{gathered}
$$

The $\bar{c}_{i j}$ differ from the usual elastic stiffness coefficients $c_{i j}$ because the $\bar{c}_{i j}$ correspond to tetragonal deformations that maintain the square symmetry $a_{1}=a_{2}{ }^{6}$ To separate $c_{11}$ from $c_{12}$ requires breaking tetragonal symmetry, but is not possible if $E$ is known only from the power series (1). However, when the phase has cubic symmetry $c_{11}=c_{33}$ and $c_{12}$ can be evaluated. In fact, $c_{12}$ can be evaluated in two ways, i.e., from $\bar{c}_{11}$ and $\overline{c_{33}}$ on the one hand and from $\bar{c}_{13}$ on the other. The correspondence of the two values is then a test of the accuracy of the power-series representation of $E$, as will be noted in Sec. IV.

A strained tetragonal state will in general be maintained by applied in-plane and out-of-plane stresses determined by the derivatives $(\partial E / \partial a)_{c}$ and $(\partial E / \partial c)_{a}$. However, stability depends also on a condition on the second derivatives of $E$, which states that the second-order differential of $E$ is always positive, i.e., that

$$
\delta^{2} E=V\left[\frac{1}{2} \bar{c}_{11}\left(\frac{\delta a}{a}\right)^{2}+\bar{c}_{13} \frac{\delta a}{a} \frac{\delta c}{c}+\frac{1}{2} \bar{c}_{33}\left(\frac{\delta c}{c}\right)^{2}\right]
$$

is greater than zero for all deformations $\delta a$ and $\delta c$. Otherwise the structure would have a tetragonal deformation that lowers the energy, so that the structure cannot be maintained by applied stresses. The condition $\delta^{2} E>0$ is then a condition on the $\bar{c}_{i j}$, namely,

$$
D \equiv \overline{c_{11}} \bar{c}_{33}-{\overline{c_{13}}}^{2}>0 \text {. }
$$

The lines along which $D=0$ can be calculated readily by finding the $c / a$ at which $D=0$ for the function $E(c / a)$ at constant $V$ and using a range of $V$ to follow the line; the analytical power series for the second derivatives of $E$ obtained from Eq. (1) are convenient for the calculation.
The slope of the EBP at the phase points can be expressed directly in terms of the elastic constants of each tetragonal or cubic phase, volume $V_{0}=c_{0} a_{0}^{2} / 2$,

$$
\left(\frac{d\left(V / V_{0}\right)}{d(c / a)}\right)_{\mathrm{EBP}}=-\frac{a_{0}}{c_{0}} \frac{\left(2 \bar{c}_{33}-\bar{c}_{13}\right)}{\left(\overline{c_{33}}+\bar{c}_{13}\right)}=-\frac{2 a_{0}}{c_{0}} \frac{\left(c_{33}-c_{13}\right)}{\left(c_{33}+2 c_{13}\right)} .
$$

If the phase point has cubic symmetry, Eq. (5) simplifies, since then $c_{11}=c_{33}, c_{12}=c_{13}$ and

$$
\left(\frac{d\left(V / V_{0}\right)}{d(c / a)}\right)_{\mathrm{EBP}}=-\frac{2 a_{0}}{c_{0}} \frac{\left(c_{11}-c_{12}\right)}{\left(c_{11}+2 c_{12}\right)}=-\frac{2 a_{0}}{c_{0}} \frac{1-2 v}{1+v},
$$

where $\nu$ is the Poisson ratio of the cubic phase $\nu=c_{12} /\left(c_{11}\right.$ $\left.+c_{12}\right)$. In the figures $V_{0}$ is chosen as the volume of the equilibrium phase of lower $E$, hence Eq. (5) or (6) applied to the other equilibrium phase has a factor of the ratio of the $V$ of the other phase to $V_{0}$ on the right-hand sides of Eqs. (5) and (6) to get the slope of the plotted EBP.

Equations (5) and (6) relate the linear elastic approximation to the EBP of a phase directly to the elastic constants for a tetragonal noncubic phase or a cubic phase, respectively. If the elastic constants of the phase are known from experiment or theory, the equations give the linear approximation to the EBP. Then the measured bulk structure of a strained epitaxial film can be compared with the linear EBP to identify the equilibrium phase of that film. This identification is especially interesting for noncubic tetragonal phases, which are predicted to exist for all transition elements, ${ }^{1,3}$ but are always metastable. Hence they cannot be made macroscopically, but may be stabilized by epitaxy, as was done in the case of bct Co. ${ }^{4}$ A comparison of Eq. (5) with Eq. (6) shows that Eq. (5) defines an effective Poisson ratio for tetragonal phases.

\section{RESULTS}

The results of calculations with the procedures and formulas of Sec. II are given in four figures and two tables. Figures 1-3 plot the EBP's of $\mathrm{V}, \mathrm{Ti}$, and $\mathrm{Sr}$ along with contours of constant $E$ on the $c / a-V / V_{0}$ tetragonal plane, where $V_{0}$ is the volume per atom of the more stable phase point; the positions of the two phase points and the saddle point are marked. The composite Fig. 4 plots the EBP's of $\mathrm{Rb}, \mathrm{Li}$, and $\mathrm{K}$ without the contour lines. The corresponding coordinates and the energy at each point referred to a zero at the more stable phase are given in Table I. Plotted in Figs. 1-3 are five contours of constant energy, i.e., two contours at $\delta E$ above the two minima, the contours through the saddle point, and the contours $\delta E$ above and below the saddle-point energy. The values chosen for $\delta E$ depend on the energy scale for each metal. The unstable region where $D<0$, which includes the saddle point, is the region between the two lines of long dashes and is shown in all four figures. Table I also gives the stationary points of $E$ for tetragonal $\mathrm{Rb}$ computed by Milstein, Marschall, and Fang ${ }^{7}$ from an empirical potential fitted to experiment.

The tetragonal elastic stiffness constants $\overline{c_{11}}, \overline{c_{13}}, \overline{c_{33}}$ are given in Table II at the phase points and also the elastic stiffness constants found in Ref. 3 for the cubic phases of each metal. When the phase is cubic, the usual tetragonal 


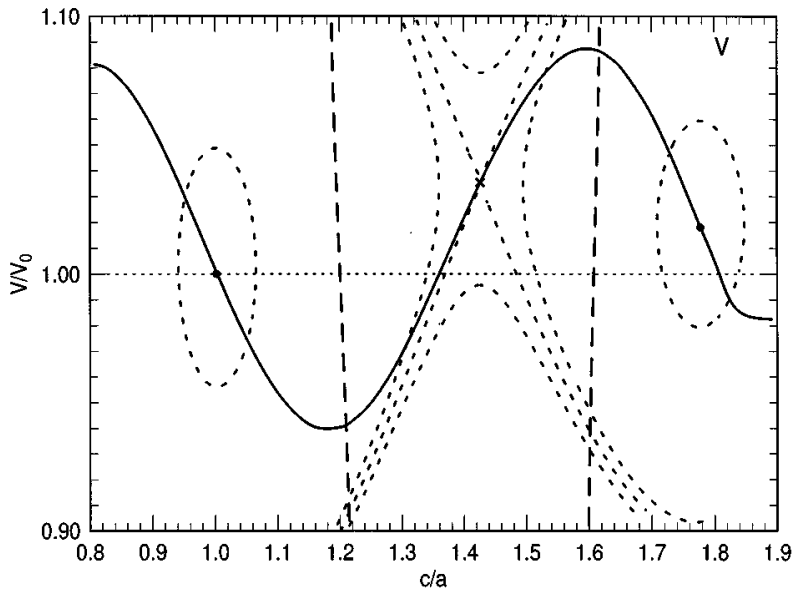

FIG. 1. EBP for $\mathrm{V}$ on the $c / a-V / V_{0}$ plane (full line), phase point $1 \mathrm{bcc}$ (full circle), volume $V_{0}=84.8 \mathrm{bohr}^{3}, E=0$; saddle point fcc (marked $\times), E=20.8 \mathrm{mRy}$; phase point $2 \mathrm{bct}$ (full circle), $E=10.0 \mathrm{mRy}$. Energy contours are drawn (short dashes) at $\delta E$ $=1 \mathrm{mRy}$ above the minima, through the saddle point, and $\pm \delta E$ from the saddle point. The unstable region is between the lines of long dashes and includes the saddle point. The coordinates for the phase and saddle points are in Table I.

elastic constants $c_{11}$ and $c_{12}$ (found in two ways) are given; note that then $c_{11}=\bar{c}_{33}$.

By symmetry, $E$ is stationary at the cubic points $(c / a$ $=1, \sqrt{2})$ on the EBP, i.e., $\partial E / \partial a=\partial E / \partial c=0$ at cubic points. However, the cubic points can be saddle points as well as minima. The possible configurations have been classified in Ref. 7 in three cases, i.e., case 1 , minimum at bcc $(c / a$ $=1$ ), saddle point at $1<c / a<\sqrt{2}$, and minimum at fcc $(c / a=\sqrt{2})$; case 2 , minimum at bct $(c / a<1)$, saddle point at bcc $(c / a=1)$, and minimum at fcc $(c / a=\sqrt{2})$; and case 3, minimum at bcc $(c / a=1)$, saddle point at fcc $(c / a=\sqrt{2})$, and minimum at bct $(c / a>\sqrt{2})$. Then $\mathrm{Rb}, \mathrm{K}, \mathrm{Li}$, and $\mathrm{Sr}$ are case $1, \mathrm{Ti}$ is case 2 , and $\mathrm{V}$ is case 3 .

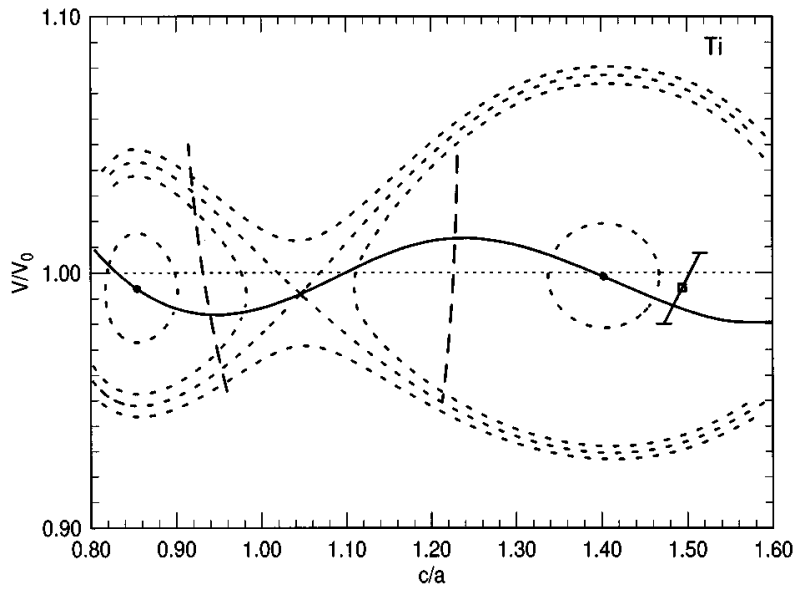

FIG. 2. EBP for Ti, stationary points, contour lines, and unstable region marked as in Fig. 1. Phase point 1 is bct at $E=1.59 \mathrm{mRy}$; the saddle point is bcc at $E=2.59 \mathrm{mRy}$; phase point 2 is fcc at $E$ $=0 \mathrm{mRy}, V_{0}=108.1 \mathrm{bohr}^{3}, \delta E=0.2 \mathrm{mRy}$. The measured strained bulk structure of epitaxial film on $\mathrm{Al}(001)$ is marked by the open square with error line (Ref. 8).

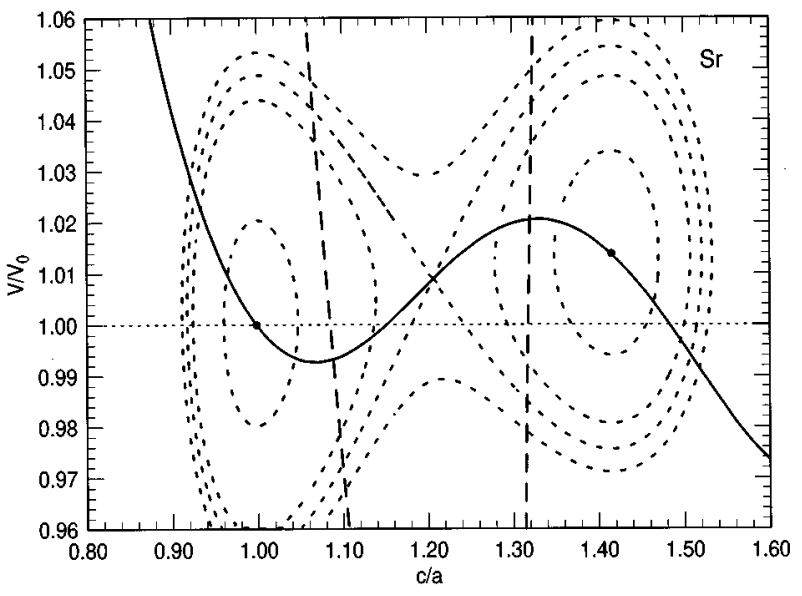

FIG. 3. EBP for Sr, stationary points, contour lines, and unstable region marked as in Fig. 1, phase point 1 is bcc at $E=0 \mathrm{mRy}$; the saddle point is bet at $E=0.383 \mathrm{mRy}$; phase point 2 is fcc at $E$ $=0.190 \mathrm{mRy}, V_{0}=319.6 \mathrm{bohr}^{3}, \delta E=0.07 \mathrm{mRy}$.

The contour lines of constant $E$, which are vertically oriented ellipses near each phase point, also appear in Ref. 3 plotted on the $c / a-V$ plane for each metal. The EBP and contour lines for $V$ are also in Ref. 4, where they are plotted on the $a / a_{0}-V / V_{0}$ plane. On this plane the contours are tilted and the bcc and fcc positions on the EBP are reversed. The contours on the $c / a-V$ plane in terms of the deviations of $c / a$ and $V$ from the values at the phase points are given by

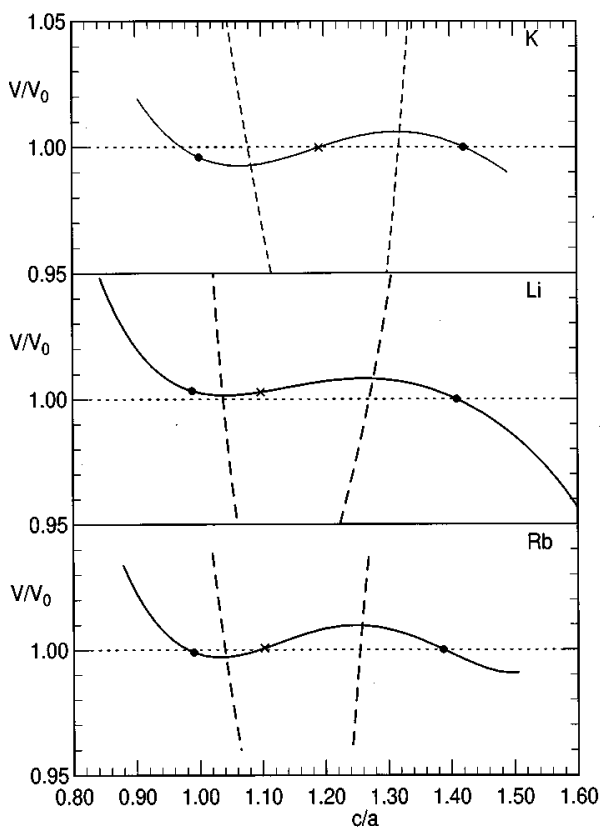

FIG. 4. EBP for $\mathrm{Rb}, \mathrm{Li}$, and $\mathrm{K}$, stationary points, and unstable region marked as in Fig. 1. Phase point 1 for $\mathrm{Rb}$ is bcc at $E$ $=0.135 \mathrm{mRy}$; the saddle point is bct at $E=0.157 \mathrm{mRy}$; phase point 2 is fcc at $E=0 \mathrm{mRy}, V_{0}=518.0 \mathrm{bohr}^{3}$. Phase point 1 for $\mathrm{Li}$ is bcc at $E=0.144 \mathrm{mRy}$; the saddle point is bct at $E=0.161 \mathrm{mRy}$; phase point 2 is fcc at $E=0 \mathrm{mRy}, V_{0}=127.7 \mathrm{bohr}^{3}$. Phase point 1 for $\mathrm{K}$ is bcc at $E=0.013 \mathrm{mRy}$; the saddle point is bet at $E=0.089 \mathrm{mRy}$, $V_{0}=430.4 \mathrm{bohr}^{3}$. 
TABLE I. Tetragonal states stationary in energy. The parameter are $(c / a)_{i},\left(V / V_{0}\right)_{i}, E_{i}, i=1,2,3$, in the tetragonal plane for states of stationary $E$; the stable phase point is $i=1$ or 3 and the metastable phase point is then $i=3$ or 1 , respectively; the saddle point is $i=2$. Note that the tetragonal lattice parameters can be found from $a=\left[2 V_{0}\left(V / V_{0}\right) /(c / a)\right]^{1 / 3}$ and $c=a(c / a) . V_{0}$ is the volume per atom of the more stable phase point in bohr $^{3}$ and $E_{i}$ is the energy in mRy with respect to the energy of the more stable phase point.

\begin{tabular}{lcccccccccc}
\hline \hline Metal & $V_{0}$ & $(c / a)_{1}$ & $\left(V / V_{0}\right)_{1}$ & $E_{1}$ & $(c / a)_{2}$ & $\left(V / V_{0}\right)_{2}$ & $E_{2}$ & $(c / a)_{3}$ & $\left(V / V_{0}\right)_{3}$ & $E_{3}$ \\
\hline $\mathrm{V}$ & 84.9 & 1.00 & 1.00 & 0.00 & 1.43 & 1.04 & 20.8 & 1.78 & 1.02 & 10.0 \\
$\mathrm{Ti}$ & 107.9 & 0.85 & 0.99 & 1.59 & 1.05 & 0.99 & 2.59 & 1.40 & 1.00 & 0.0 \\
$\mathrm{Rb}^{\mathrm{a}}$ & 518.0 & 0.99 & 0.999 & 0.135 & 1.10 & 1.001 & 0.157 & 1.39 & 1.00 & 0.0 \\
$\mathrm{Rb}^{\mathrm{b}}$ & & 1.00 & 0.997 & -0.042 & 1.22 & 0.999 & 0.041 & 1.41 & 1.00 & 0.0 \\
$\mathrm{~K}$ & 430.4 & 1.00 & 0.996 & 0.013 & 1.19 & 1.000 & 0.089 & 1.42 & 1.00 \\
$\mathrm{Li}$ & 127.7 & 0.99 & 1.003 & 0.144 & 1.10 & 1.003 & 0.161 & 1.41 & 1.00 \\
$\mathrm{Sr}$ & 319.6 & 1.00 & 1.000 & 0.000 & 1.21 & 1.009 & 0.383 & 1.42 & 1.014 \\
\hline \hline
\end{tabular}

${ }^{\mathrm{a}}$ This work.

${ }^{b}$ From Ref. 7.

$$
\begin{aligned}
& E-E_{\min } \simeq c_{1}\left(\frac{\delta c / a}{c / a}\right)^{2}+c_{2}\left(\frac{\delta c / a}{c / a}\right)\left(\frac{\delta V}{V}\right)+c_{3}\left(\frac{\delta V}{V}\right)^{2}, \\
& c_{1} \equiv(V / 18)\left(\bar{c}_{11}-4 \bar{c}_{13}+4 \bar{c}_{33}\right), \\
& c_{2} \equiv-(V / 9)\left(\bar{c}_{11}-\bar{c}_{13}-2 \bar{c}_{33}\right), \\
& c_{3} \equiv(V / 18)\left(\bar{c}_{11}+2 \bar{c}_{13}+\bar{c}_{33}\right) .
\end{aligned}
$$

For cubic symmetry $c_{2}$ vanishes; even for the bct phases of Ti and $\mathrm{V}, c_{2}$ is much smaller than $c_{1}$ and $c_{3}$, so the contours are still nearly vertical. Formula (7) for the case of cubic symmetry with coefficients in terms of the $c_{i j}$ is given in Ref. 2.

\section{DISCUSSION}

The principal result found here is the EBP between the phase points, which shows the effects of isotropic epitaxial strain on equilibrium phases. The EBP provides a basic elas- tic response of a material in tetragonal structure to a particular strain, one that is available experimentally, including the interesting case of negative strain in the plane. The EBP's are found here from first principles in a well-defined approximation with errors of known magnitude and include nonlinear effects. These EBP can then be compared directly with the strained bulk structures determined by QLEED for epitaxial films. This comparison is illustrated for strained epitaxial Ti films on $\mathrm{Al}(001),{ }^{8}$ where the QLEED point and its error limits (from the uncertainty in the bulk value of $c$ ) are shown in Fig. 2 to agree well with the calculated EBP. The $V_{0}$ used in evaluating $V / V_{0}$ for the QLEED point is the measured hcp Ti value $V_{0}^{\mathrm{hcp}}=119.2 \mathrm{bohr}^{3}$, which is close to the fcc value.

The presence on the EBP of an inherently unstable section separating strained fcc Ti from strained bct Ti is an important result of the theory. Since the QLEED point within its error limit lies on the fcc part of the EBP, the epitaxial film must be strained fcc Ti. This result is particularly interesting because fcc $\mathrm{Ti}$ does not appear on the usual pressure-

TABLE II. Elastic constants of stable and metastable phases: The tetragonal elastic stiffness constants $\bar{c}_{11}, \bar{c}_{13}, \bar{c}_{33}$ in Mbar for phase 1 at stationary point 1 of Table I and for phase 2 at stationary point 3 of Table I, $c_{11}, c_{12}, c_{33}$ are the usual elastic constants for tetragonal structures. For cubic phases $c_{11}=c_{33}=\bar{c}_{33}$; hence, from Eq. (2), $c_{12}$ is found in two ways: $c_{12}^{(1)}=\left(\bar{c}_{11} / 2\right)-\bar{c}_{33}$ and $c_{12}^{(2)}=\bar{c}_{13} / 2$. Note that $1 \mathrm{mRy} / \mathrm{bohr}^{3}=0.14711$ Mbar.

phase 1

phase 2

\begin{tabular}{lcccccccccccc} 
Metal & $c / a$ & $\overline{c_{11}}$ & $\bar{c}_{13}$ & $\bar{c}_{33}$ & $c_{12}^{(1)}$ & $c_{12}^{(2)}$ & $c / a$ & $\bar{c}_{11}$ & $\bar{c}_{13}$ & $\bar{c}_{33}$ & $c_{12}^{(1)}$ & $c_{12}^{(2)}$ \\
\hline $\mathrm{V}^{\mathrm{a}}$ & 1.00 & 10.26 & 3.27 & 3.60 & 1.53 & 1.63 & 1.78 & 11.47 & 2.08 & 4.57 \\
$\mathrm{~V}^{\mathrm{b}}$ & & & & 2.88 & 1.36 & & & & & & \\
$\mathrm{Ti}^{\mathrm{a}}$ & 0.85 & 5.05 & 2.18 & 1.45 & & & 1.40 & 5.52 & 2.38 & 1.59 & 1.17 & 1.19 \\
$\mathrm{Ti}^{\mathrm{b}}$ & & & & & & & & & 1.48 & 1.21 \\
$\mathrm{Rb}^{\mathrm{a}}$ & 0.99 & 0.160 & 0.074 & 0.043 & 0.037 & 0.037 & 1.39 & 0.163 & 0.075 & 0.048 & 0.034 & 0.038 \\
$\mathrm{Rb}^{\mathrm{b}}$ & & & & 0.045 & 0.038 & & & & 0.046 & 0.040 \\
$\mathrm{~K}^{\mathrm{a}}$ & 1.00 & 0.220 & 0.100 & 0.059 & 0.051 & 0.048 & 1.42 & 0.214 & 0.097 & 0.062 & 0.046 & 0.049 \\
$\mathrm{~K}^{\mathrm{b}}$ & & & & 0.061 & 0.049 & & & & & 0.057 & 0.050 \\
$\mathrm{Li}^{\mathrm{a}}$ & 0.99 & 0.633 & 0.296 & 0.165 & 0.151 & 0.148 & 1.41 & 0.636 & 0.280 & 0.178 & 0.140 & 0.140 \\
$\mathrm{Li}^{\mathrm{b}}$ & & & & 0.170 & 0.147 & & & & & 0.169 & 0.143 \\
$\mathrm{Sr}^{\mathrm{a}}$ & 1.00 & 0.678 & 0.283 & 0.194 & 0.145 & 0.141 & 1.42 & 0.698 & 0.295 & 0.202 & 0.147 & 0.147 \\
$\mathrm{Sr}^{\mathrm{b}}$ & & & & 0.206 & 0.136 & & & & 0.189 & 0.153 \\
\hline
\end{tabular}

${ }^{\text {a This work. }}$

${ }^{\mathrm{b}}$ From Ref. 3. 
temperature phase diagram. Note that the theory gives directly the possible states of epitaxially strained fcc $\mathrm{Ti}$, including any nonlinear elastic behavior of the crystalline phases. This comparison of measured structure with the theoretical EBP replaces the previous analysis, which assumed constant elastic stiffness coefficients and attempted to estimate the elastic coefficients of the cubic phases. ${ }^{8}$ The use of linear elastic relations for identification of the equilibrium phase is compared to the use of the EBP for that identification in the case of Co in Ref. 9, where tetragonal states and the EBP are plotted on the $a-d$ plane, where $d$ is the layer spacing.

These first-principles calculations, which do not use empirical information, have an advantage over empirical potentials fitted to measurements since these calculations are as good for highly strained or even unstable states, which are not accessible to measurement, as they are for slightly strained equilibrium states. Thus they provide a test of calculations based on empirical potentials, such as the potential used for $\mathrm{Rb}$ in Ref. 7. The comparison in Table I shows that the $c / a$ values of the saddle point differ by $10 \%$, that the minimum energy in Ref. 7 is at the bcc structure, rather than the fcc structure found here, and that the energy separation of the equilibrium phases in Ref. 7 has both a sign and a magnitude different (smaller) from what is calculated here.

The power-series representation of the tetragonal energies shows some defects, e.g., the cubic points deviate from $\mathrm{cla}$ $=1$ or $\sqrt{2}$ in Table I and the two values of $c_{12}$ do not agree well in some cases. These defects are in the representation of the results of LDA calculations. The most serious defect is in the saddle point of $\mathrm{Ti}$, which is bcc, but the power series finds the saddle point at $c / a=1.05$ rather than 1 . A recalculation of the energy $E$ of Ti directly with WIEN95 finds that the power series has missed an asymmetry in $E$ around the saddle point that shifts the position of the maximum. The direct calculation finds the maximum at $c / a=1$, as it should be; it also verifies the minimum of fcc-Ti at $c / a=1.40$. The deviation from symmetry at fcc $\mathrm{Ti}$ and all other cubic phases is thus no more than $1 \%$, except for fcc Rb, where the deviation is $1.7 \%$. In comparison to experiment, all the calculated elastic stiffness coefficients are too large by at least $10 \%$ and the volumes per atom are too small by $5-10 \%$. These discrepancies from experiment are defects of the assumptions of the band calculations, i.e., of the LDA with semirelativistic corrections.

Despite the deviations from experiment, which may be reduced in subsequent calculations by more accurate formulation of the electronic-structure equations, these results are of immediate practical value in interpreting measured film structures and of conceptual value in providing a sharp distinction between, for example, a tetragonally strained bcc phase and a tetragonally strained fcc phase. A generalization of the tetragonal results to other structures suggests a different type of phase diagram in which the various equilibrium phases, stable and metastable, are points in a parameter space that has structural parameters as coordinates. The present results suggest that each phase point is surrounded by a region of strained states and the regions are embedded in and separated by a continuous matrix of inherently unstable states that cannot be stabilized by applied stresses. Such a generalization for structures with considerable symmetry, such as the tetragonal structure, seems calculable by present codes.

\section{ACKNOWLEDGMENTS}

Thanks are due to M. Scheffler of the Fritz-Haber Institute for discussion and encouragement and to K. Schwarz and P. Mohn of the Technical University of Vienna for information about their energy calculations.
${ }^{1}$ P. J. Craievich, M. Weinert, J. M. Sanchez, and R. E. Watson, Phys. Rev. Lett. 72, 3076 (1994); Phys. Rev. B 55, 787 (1997).

${ }^{2}$ T. Kraft, P. M. Marcus, M. Methfessel, and M. Scheffler, Phys. Rev. B 48, 5886 (1993).

${ }^{3}$ V. L. Sliwko, P. Mohn, K. Schwarz, and P. Blaha, J. Phys.: Condens. Matter 8, 799 (1996).

${ }^{4}$ P. Alippi, P. M. Marcus, and M. Scheffler, Phys. Rev. Lett. 78, 3892 (1997).

${ }^{5}$ The coefficient $A_{43}$ for Ti in Ref. 3 should read -0.54515791 $\times 10^{-2}$ instead of $-0.54515719 \times 10^{-2} \mathrm{P}$. Mohn (private communication). Reference 3 also includes eight-figure coefficients for $\mathrm{Ca}$, but eleven figures in the coefficients are needed to get accurate results; they can be obtained from the authors of Ref. 3 .

${ }^{6}$ The relations between the $\bar{c}_{i j}$ and the $c_{i j}$ in Eq. (2) are derived by comparing second derivatives of $E$ obtained in two ways. One way uses Eq. (2) and treats $E$ as a function of the two variables $a$ and $c$. The second way treats $E$ as a function of three orthogonal variables $a_{1}$ and $a_{2}$, the two in-plane lattice constants, and $c$, the out-of-plane lattice constant, and takes second derivatives with respect to $a_{1}$ or $a_{2}$ or the mixed second derivative with respect to $a_{1}$ and $c$, all at $a_{1}=a_{2}=a$. The second way generates the usual $c_{i j}$.

${ }^{7}$ Frederick Milstein, Jochen Marschall, and Huei Eliot Fang, Phys. Rev. Lett. 74, 2977 (1995).

${ }^{8}$ S. K. Kim, F. Jona, and P. M. Marcus, J. Phys.: Condens. Matter 8, 25 (1996).

${ }^{9}$ F. Jona and P. M. Marcus, Surf. Rev. Lett. (to be published). 